\title{
Análise de Desempenho da Codificação Wavelet em Canais Seletivos em Frequência
}

\author{
Lucas Costa P. Cavalcante, Aluisio Igor F. Rêgo, Marcelo Augusto C. Fernandes, Luiz Felipe de Q. Silveira
}

\begin{abstract}
Resumo-A codificação wavelet surgiu como uma técnica de codificação de canal alternativa para minimizar os efeitos nocivos dos canais de comunicação sem fio caracterizados pelo desvanecimento. Diferente de técnicas clássicas de codificação, nas quais o ganho de codificação surge através da adição de uma redundância controlada a informação, a robustez conseguida pela codificação wavelet contra os efeitos do desvanecimento ocorre em função de uma diversidade temporal gerada sobre a informação transmitida. Até o momento, o desempenho da codificação wavelet foi investigado apenas em canais de comunicação sujeito a desvanecimento plano variante no tempo. Este trabalho avalia o desempenho da codificação wavelet, em termos de probabilidade de erro de bit, sobre canais de comunicação com desvanecimento Rayleigh variante no tempo e seletivo em frequência. Através de simulação computacional, apresenta-se neste trabalho resultados numéricos que demonstram a eficiência dessa técnica no combate à interferência entre símbolos, característica destes cenários de comunicação.
\end{abstract}

Palavras-Chave-Sistemas de comunicação sem fio, Codificação wavelet, Diversidade temporal, Desvanecimento Rayleigh, Desvanecimento Seletivo em Frequência.

Abstract-Wavelet coding has emerged as an alternative coding technique to minimize the fading effects of wireless channels. Unlike classical coding techniques, in which the coding gain arises through the controlled addition of redundant information, the robustness achieved by wavelet encoding against the fading effects occurs due to a time diversity created over the transmitted information. So far, the performance of wavelet coding has been investigated only in communication channels subject to time-varying flat fading. This paper evaluates the performance of wavelet coding, in terms of bit error probability, over time-varying, frequency-selective multipath Rayleigh fading channels. Through computer simulation, this paper presents numerical results that demonstrate the effectiveness of this technique in combating inter-symbolic interference, characteristic of these communication scenarios.

Keywords-Wireless Communications, Wavelet Coding, Time Diversity, Rayleigh Fading, Frequency Selective Fading.

\section{INTRODUÇÃO}

Nos sistemas de comunicação sem fio, o sinal pode percorrer o meio entre as antenas transmissoras e receptoras por mais de um percurso. A presença de múltiplos percursos de propagação se deve à fatores como espalhamento e refração atmosféricas, ou reflexões em prédios e outros obstáculos. Neste ambiente de comunicação, os sinais que chegam por diferentes caminhos terão atenuações e atrasos distintos. Além disso, os percursos podem ser constantemente alterados com a mudança da geometria do canal ou pelo movimento

Lucas Costa P. Cavalcante, Aluisio Igor F. Rêgo, Marcelo Augusto C. Fernandes, Luiz Felipe de Q. Silveira estão com o Programa de Pós-Graduação em Engenharia Elétrica e de Computação, Universidade Federal do Rio Grande do Norte, Natal, Brasil, E-mails: \{lucas,aluisio,mfernandes,lfelipe\} @ dca.ufrn.br relativo entre as antenas. Esses fenômenos caracterizam o desvanecimento por múltiplos percursos, que geralmente afeta o sinal de duas maneiras: atenuação variante no tempo e dispersão. Em particular, a dispersão do sinal no tempo pode ocasionar interferência intersimbólica (ISI), vista no domínio da frequência como uma seletividade no canal de comunicação [1]- [5].

As características do canal sem fio claramente impõem degradação na qualidade do sinal de comunicação. A questão "Como minimizar os efeitos causados por esse tipo de enlace?", conduz uma investigação por técnicas de transmissão que possam aumentar a confiabilidade dos sistemas de comunicação digital [6]. Trabalhos recentes indicam que as matrizes de coeficientes wavelets podem ser empregadas nesta tarefa [7]-[9]. De fato, algumas de suas propriedades, como o comportamento ortogonal entre suas linhas, podem proporcionar ganhos de diversidade a um custo computacional baixo, tornando-as adequadas para serem empregadas em técnicas de codificação para canais sem fio.

A codificação wavelet foi inicialmente proposta em [8], sendo referenciada posteriormente por [7] e analisada em diversos trabalhos [9]- [13]. Apesar dos promissores resultados na tarefa de combate aos efeitos do desvanecimento plano, investigações adicionais sobre o uso da codificação wavelet para combater desvanecimento seletivo em frequência não tem sido reportado na literatura.

Neste artigo, investigamos o desempenho da codificação wavelet sob essas condições de desvanecimento. Acredita-se que a avaliação de desempenho desenvolvida nesse trabalho pode ressaltar a robustez da codificação wavelet ao lidar com ambientes severos de comunicação e assim, estimular novas pesquisas nesse tema. Apresentamos resultados de simulação em quatro situações distintas de comunicação, incluindo uma condição de seletividade severa. Como referência de desempenho, consideramos um sistema de comunicação com codificação wavelet sujeito a desvanecimento plano e um sistema não-codificado, com eficiência espectral idêntica ao dos sistemas codificados.

O restante deste trabalho está organizado como segue: A Seção II aborda os fundamentos da codificação wavelet e os algoritmos de codificação e decodificação. A Seção III descreve o modelo do sistema de comunicação investigado, incluindo detalhes do esquema de modulação empregado, do canal de transmissão, e da recepção com demodulação por métrica Euclidiana. A Seção IV apresenta os resultados das simulações e a Seção V contém as conclusões do trabalho. 


\section{CODIFICAÇÃo WAVELET}

A técnica de codificação descrita neste trabalho explora as propriedades das Matrizes de Coeficientes Wavelets (MCW). Essas matrizes possuem linhas arbitrariamente longas que são ortogonais entre si - e que assim se mantêm, mesmo quando deslocadas e adicionadas. É através delas que a informação representada por um bit de informação espalha-se por todo o sinal transmitido, e ao mesmo tempo faz com que uma pequena parte do sinal contenha informação sobre o todo. A ortogonalidade entre as linhas da MCW é usada pelo decodificador wavelet para estimar, por correlação, cada bit de informação que encontra-se espalhado no sinal recebido. O mecanismo de espalhamento da informação no tempo, antes da transmissão e, o recolhimento dela no receptor, contribui para melhorar a robustez do sistema de comunicação à combinação de efeitos do desvanecimento variante no tempo e de ruído localizado.

Uma das vantagens proporcionadas pelo emprego da codificação wavelet é a possibilidade que esta oferece de aumentar o ganho de diversidade temporal, melhorando o desempenho do sistema de comunicações frente aos efeitos nocivos do canal com desvanecimento Rayleigh. A obtenção de melhores ganhos de diversidade é proporcionada pelo emprego de uma matriz wavelet de maiores dimensões, que implica no espalhamento da informação de cada bit sobre um número maior de símbolos wavelets.

\section{A. Matrizes de Coeficientes Wavelet}

Considere a matriz $\mathbf{A}=\left(a_{k}^{s}\right)$ com $m \geq 2$ linhas e $m g$ colunas denotada por:

$$
\mathbf{A}=\left(\begin{array}{cccc}
a_{0,0} & a_{0,1} & \cdots & a_{0, m g-1} \\
a_{1,1} & a_{1,1} & \cdots & a_{1, m g-1} \\
\vdots & \vdots & \ddots & \vdots \\
a_{m-1,0} & a_{m-1,1} & \cdots & a_{m-1, m g-1}
\end{array}\right)
$$

com elementos no conjunto dos números complexos. A matriz A é denominada de matriz wavelet de posto $m$ e gênero $g$ se as seguintes condições forem satisfeitas:

$$
\begin{gathered}
\sum_{k=0}^{m g-1} a_{k}^{s}=\delta_{s, 0}, \quad 0 \leq s \leq m-1, \\
\sum_{k=0}^{m g-1} a_{\left[k+m r^{\prime}\right]}^{s^{\prime}} \bar{a}_{[k+m r]}^{s}=m \delta_{s^{\prime}, s} \delta_{r^{\prime}, r}, \\
0 \leq s^{\prime}, s \leq m-1 ; 0 \leq r^{\prime}, r \leq m-1,
\end{gathered}
$$

em que $[k+m r]$ é usado para denotar a operação $k+m r$ módulo $m g, \bar{a}$ é o conjugado complexo de $a$ e $\delta_{x, y}$ é o símbolo de Kronecker, definido por:

$$
\delta_{x, y}= \begin{cases}1 & \text { se } x=y \\ 0 & \text { caso contrário. }\end{cases}
$$

A Equação (3) estabelece que as linhas de uma MCW de posto $m$ têm comprimento igual a $\sqrt{m}$ e são mutuamente ortogonais, mesmo quando deslocadas de si por um múltiplo de $m$. Além disso, ela indica que cada linha da MCW é ortogonal a uma cópia de si mesma deslocada por um múltiplo de $m$. A Equação (2) assegura que a soma dos elementos da primeira linha da matriz é igual ao posto da matriz wavelet, enquanto que a soma dos elementos das demais linhas é igual a zero.

\section{B. Matrizes Wavelet Utilizadas na Codificação}

Uma matriz wavelet plana possui a propriedade de que todos os seus elementos têm o mesmo valor absoluto. Ela é denominada matriz wavelet real plana quando todos os seus elementos são reais. E quando todos os seus elementos estão normalizados em \pm 1 , então valem as seguintes condições, modificadas a partir das Equações (2) e (3) [8]:

$$
\begin{gathered}
\sum_{k=0}^{m g-1} a_{k}^{s}=m \sqrt{g} \delta_{s, 0}, \\
\sum_{k=0}^{m g-1} a_{\left[k+m r^{\prime}\right]}^{s^{\prime}} \bar{a}_{[k+m r]}^{s}=m g \delta_{s^{\prime}, s} \delta_{r^{\prime}, r},
\end{gathered}
$$

A matriz wavelet utilizada neste trabalho é plana, com elementos normalizados em \pm 1 , e tem dimensão $2 \times 128$.

\section{Algoritmo de Codificação Wavelet}

No processo de codificação, a sequência de bits de informação $x_{n} \in\{+1,-1\}$ é inicialmente dividida em $m$ sequências paralelas, definidas por $X_{p m+j}:=$ $\left\{x_{p m+j}\right\}_{p \in \mathbb{Z}}, 0 \leq j<m$, como pode ser observado na Figura 1(a). A $j$-ésima sequência paralela $X_{p m+j}$ é então codificada por um banco de registradores de deslocamento, denotado pelo bloco $M C W_{j}$ e detalhado na Figura 1(b). No instante de tempo $n=p m+q$, em que $p \in\{0,1,2,3,4, \ldots\}$ e $q \in\{0,1, \ldots, m-1\}$, o j-ésimo bloco $M C W_{j}$ do codificador wavelet gera o símbolo $y_{p m+q}^{j}$, denominado sub-símbolo wavelet.

Cada banco $\mathrm{MCW}_{j}$ é constituído por $m$ registradores de deslocamento, denotados $\mathrm{REG}_{q}$, cada um deles com $g$ células de memória, conforme ilustrado na Figura 1(b). No processo de codificação wavelet, os bits inseridos em cada um destes registradores são ponderados por coeficientes da $j$-ésima linha da MCW. Assim, os $m g$ coeficientes da $j$-ésima linha da MCW são distribuídos em $m$ grupos de $g$ coeficientes equi-espaçados, de tal forma que o $q$-ésimo grupo é formado pelos coeficientes que ponderam as células do registrador $\mathrm{REG}_{q}$ do $j$-ésimo banco.

A cada instante de tempo $n=p m+q, m$ sub-símbolos wavelet $y_{p m+q}^{j}, 0 \leq j \leq m-1$, gerados simultaneamente pelo q-ésimo registrador de cada um dos $m$ bancos $\mathrm{MCW}_{j}$, são disponibilizados na saída do codificador wavelet. A partir da Figura 1(b), pode-se observar que o sub-símbolo wavelet $y_{p m+q}^{j}$, gerado no instante de tempo $n=p m+q$, pelo $q$-ésimo registrador do banco $\mathrm{MCW}_{j}$, é dado por:

$$
y_{p m+q}^{j}=\sum_{t=0}^{g-1} a_{l m+q}^{j} x_{(p-l) m+j} .
$$

Como existem $\mathrm{mg}$ elementos de memória em cada banco de registradores de deslocamento, cada bit de entrada pode 


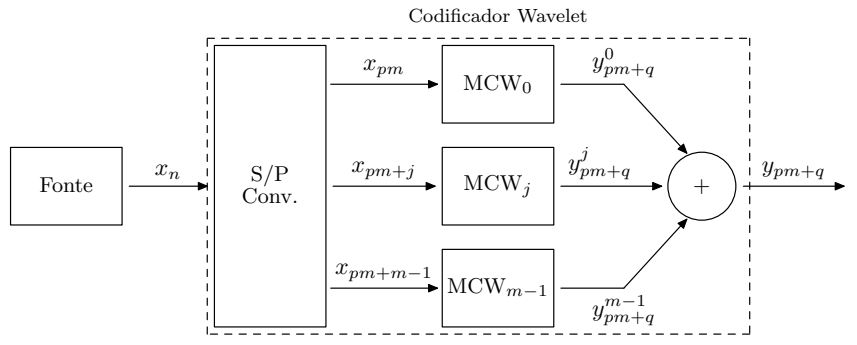

(a) Estrutura geral do codificador wavelet.

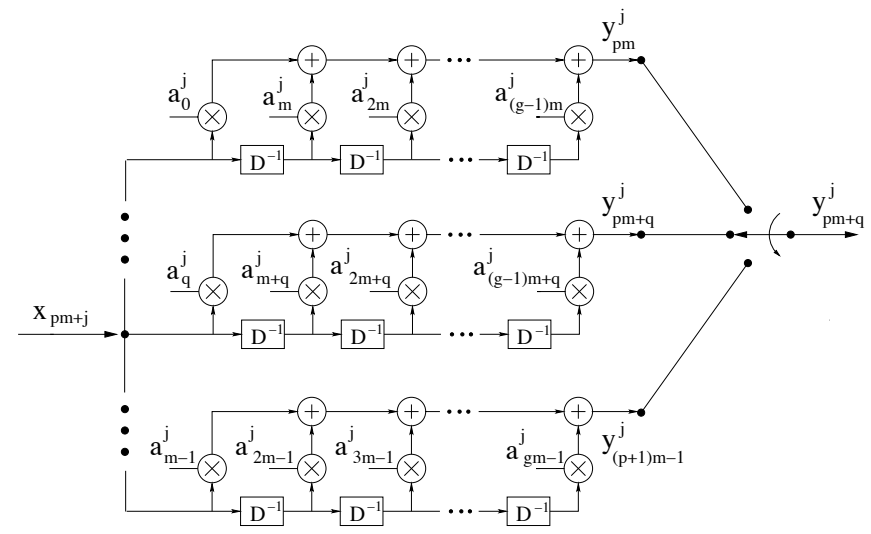

(b) Detalhamento do bloco $\mathrm{MCW}_{\mathrm{j}}$.

Fig. 1. Codificador wavelet formado por uma MCW de dimensão $m \times m g$ $\mathrm{O}$ bloco $\mathrm{WCM}_{\mathrm{j}}$ é especificado pela $j$-ésima linha da $\mathrm{MCW}$.

afetar no máximo $m g$ sub-símbolos wavelet. Finalmente, os $m$ sub-símbolos wavelet com o mesmo índice de tempo $n=$ $p m+q$ são então adicionados, conforme ilustrado na Figura 1(a), e o símbolo wavelet resultante é dado por:

$$
y_{p m+q}=\sum_{j=0}^{m-1} \sum_{l=0}^{g-1} a_{l m+q}^{j} x_{(p-l) m+j} .
$$

\section{Decodificação Wavelet}

$\mathrm{Na}$ recepção, a sequência de bits de informação $x_{n}$ pode ser recuperada a partir da sequência de símbolos $y_{n}$ recebida utilizando-se um banco de $m$ correlatores de comprimento $m g$, casados com as $m$ linhas da MCW utilizada na codificação wavelet. Admitindo ausência de ruído, a saída do correlator $z^{j}, j \in\{0,1, \ldots, m-1\}$, casado com a linha $a^{j}$ da MCW, no instante de tempo $i=m(g+p)-1$, em que $p \in \mathbb{Z}$, é dada por:

$$
\begin{aligned}
z_{i}^{j} & =\sum_{k=0}^{m g-1} a_{m g-1}^{j} y_{i-k} \\
& =\sum_{k=0}^{m g-1} \sum_{j^{\prime}=0}^{m-1} \sum_{l=0}^{g-1} a_{k}^{j}\left(a_{k-l m}^{j^{\prime}} x_{j^{\prime}+l m+i-(m g-1)}\right) \\
& \left.=x_{j+i-(m g-1)}\right) \sum_{k=0}^{m g-1} a_{k}^{j} a_{k}^{j} \\
& =m g x_{j+i-(m g-1)}
\end{aligned}
$$

De forma geral, levando-se em consideração a interferência causada pelo canal de comunicação sobre os símbolos wavelet transmitidos, assume-se estimativas dos bits dadas por $\hat{x}_{j+i-(m g-1)}=\operatorname{sgn}\left(z_{i}^{j}\right)$.

A simplicidade computacional do processo de decodificação é uma das principais vantagens da codificação wavelet [7].

\section{Modelo do Sistema}

A Figura 2 apresenta o modelo discreto em banda base do sistema de comunicação avaliado, no qual a cada instante $n$ uma sequência de bits, $x_{n}$, é transmitida. Os subsistemas que compõem o modelo serão detalhados na seqüência.

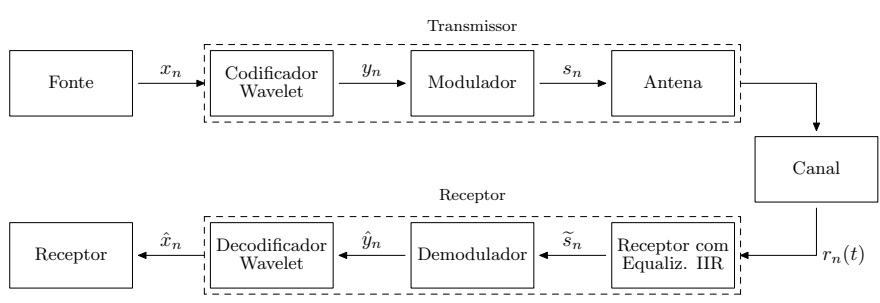

Fig. 2. Diagrama de blocos do sistema com codificação wavelet.

\section{A. Modulação}

O ganho de diversidade proporcionado pela codificação wavelet é influenciado diretamente pelo número de colunas da MCW utilizada, de tal forma que quanto maior for esse número, maior será o espalhamento temporal conseguido pela codificação. Por outro lado, o tamanho do alfabeto de símbolos gerados na codificação é igual a $m g+1$, de acordo com a Equação (8). Portanto, o emprego de matrizes wavelets de maiores dimensões pode ocasionar um acúmulo de pontos na constelação de sinais utilizada no esquema de modulação, podendo comprometer o desempenho do sistema, já que cada símbolo wavelet precisaria ser mapeado em um sinal da constelação. Assim, para viabilizar a obtenção de melhores ganhos de diversidade por parte dos sistemas que empregam a codificação wavelet é fundamental, durante o projeto do esquema de modulação, dispor de ferramentas e métodos eficientes na busca por bons esquemas de modulação.

O esquema de modulação adotado foi projetado em [9], por meio de um Algoritmo Genético (AG). Compõem esse esquema de modulação a constelação 11-PSK não-convencional ilustrada na Figura 3 e a Tabela I, utilizada para mapear os símbolos codificados nos sinais da constelação [9].

Neste trabalho, a sequência de bits, $x_{n}$, é codificada por uma matriz MCW $2 \times 128$. A cada instante de tempo $n=p m+q$, um símbolo wavelet $y_{n}$, definido pela Equação (8), é gerado na saída do codificador wavelet e modulado por um sinal $s_{n}$.

\section{B. Canal de Transmissão}

As flutuações aleatórias do sinal recebido devido ao desvanecimento podem ser modeladas, de forma discreta, por 


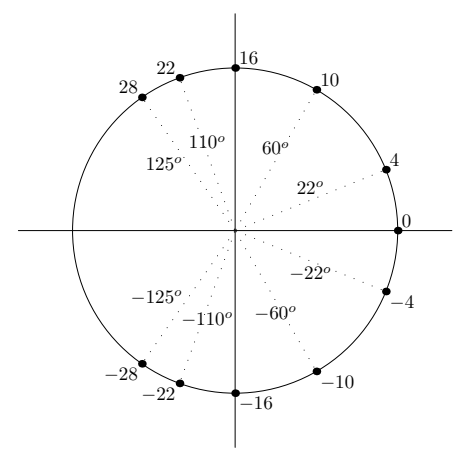

Fig. 3. Constelação 11-PSK para a MCW 2x128.

TABELA I

QUANTIZAÇÃO PARA MCW 2X128.

\begin{tabular}{|c|c|}
\hline Conjunto Truncado & Símbolo Representativo \\
\hline$\{0\}$ & 0 \\
$\{2,4,6\}$ & 4 \\
$\{-2,-4,-6\}$ & -4 \\
$\{8,10,12\}$ & 10 \\
$\{-8,-10,-12\}$ & -10 \\
$\{14,16,18\}$ & 16 \\
$\{-14,-16,-18\}$ & -16 \\
$\{20,22,24\}$ & 22 \\
$\{-20,-22,-24\}$ & -22 \\
$\{26,28,30, \ldots, 128\}$ & 28 \\
$\{-26,-28,-30, \ldots,-128\}$ & -28 \\
\hline
\end{tabular}

um canal com resposta ao impulso, $h_{n}$, expressa por

$$
h_{n}=\sum_{j=0}^{L-1} p_{j} c_{j, n} \delta_{n-\tau_{j}},
$$

em que $L$ é o comprimento do canal e $p_{j}$ e $\tau_{j}$ representam o $j$-ésimo ganho e o atraso de cada percurso do canal $h_{n}$, respectivamente. Como os componentes dos múltiplos percursos de propagação do sinal surgem em função de uma grande quantidade de reflexões e refrações a partir de diversas superfícies, a partir do teorema do limite central, $c_{j, n}$ pode ser modelada como uma variável complexa de um processo aleatório Gaussiano em $n$ associado ao $j$-ésimo percurso. Assumimos ainda que $c_{j, n}$ é um processo aleatório Estacionário no Sentido Amplo (WSS) e tem Espalhamento Descorrelacionado (US) [14], [15]. Esse modelo de canal pode ser estendido para contemplar seletividade em frequência, fazendo com que a influência do símbolo transmitido num instante $n$ alastre-se por sucessivos instantes $n-\tau_{j}$, com $\tau_{j} \geq 0$. Esse esquema implica na interferência intersimbólica da mensagem original.

A partir da Equação (10), o sinal de saída do canal, $r_{n}$ pode ser expresso por

$$
r_{n}=\sum_{j=0}^{L-1} p_{j} c_{j, n} s_{n-\tau_{j}}+\eta_{n},
$$

em que $\eta_{n}$ representa o Ruído Aditivo Gaussiano Branco no instante $n$.

\section{Demodulação}

$\mathrm{Na}$ recepção, assume-se o conhecimento do estado do canal para atribuir a um filtro IIR os pesos adequados, correspondente aos percursos e seus respectivos ganhos, no processo de inversão dos efeitos do desvanecimento causados pelo canal seletivo.

Seja $r_{n}$ o sinal na entrada do receptor após os efeitos do canal sobre a mensagem transmitida, a saída $\tilde{s}_{n}$ do receptor, que será enviada ao demodulador, pode ser dada por:

$$
\tilde{s}_{n}=\frac{1}{p_{0} c_{0, n}} r_{0}-\sum_{j=1}^{L-1} \frac{p_{j} c_{j, n}}{p_{0} c_{0, n}} r_{n-\tau_{j}},
$$

em que $p_{j} c_{n, j}$ corresponde ao produto entre os perfis de potência por atraso e as realizações do canal relacionadas, conforme descrito na Seção III-B. Na sequência, os sinais recebidos são demodulados por métrica Euclidiana.

De posse das estimativas dos sinais demodulados $\hat{s}_{n}$, a Equação (12) pode ser reformulada por:

$$
\tilde{s}_{n}=\frac{1}{p_{0} c_{0, n}} r_{0}-\sum_{j=1}^{L-1} \frac{p_{j} c_{j, n}}{p_{0} c_{0, n}} \hat{s}_{n-\tau_{j}},
$$

em que $\hat{s}_{n-\tau_{j}}$ representa o sinal demodulado no instante $n-\tau_{j}$.

Por fim, cada sinal demodulado $\hat{s}_{n}$ é mapeado na estimativa de símbolo wavelet $\hat{y}_{n}$ que o sinal representa. Esses símbolos são enviados ao decodificador wavelet para serem decodificados de acordo com o processo descrito na Seção II-D.

\section{EXPERIMENTOS E RESULTADOS}

O sistema foi analisado sob quatro configurações de canal distintas, apresentadas na Tabela II, variando-se os perfis de intensidade de múltiplos percursos. Os valores atribuídos a cada um dos percursos foram escolhidos arbitrariamente e todos os perfis foram normalizados em potência.

\section{TABELA II}

PERFIS DE CANAIS UTILIZADOS NAS SIMULAÇÕES

\begin{tabular}{|l|l|}
\hline canal 1 & $h_{n}=c_{0, n} \delta_{n}$ \\
\hline canal 2 & $h_{n}=0,9923 c_{0, n} \delta_{n}+0,1240 c_{1, n} \delta_{n-1}$ \\
\hline canal 3 & $h_{n}=0,9631 c_{0, n} \delta_{n}+0,2408 c_{1, n} \delta_{n-1}$ \\
& $+0,1204 c_{2, n} \delta_{n-2}$ \\
\hline canal 4 & $h_{n}=0,8677 c_{0, n} \delta_{n}+0,4339 c_{1, n} \delta_{n-1}$ \\
& $+0,2169 c_{2, n} \delta_{n-2}+0,1085 c_{3, n} \delta_{n-3}$ \\
\hline
\end{tabular}

A Figura 4 ilustra as curvas de taxa de erro de bit (BER) em relação a $E_{b} / N_{0}$ do sistema simulado com codificação wavelet, assim como a curva de desempenho de um sistema BPSK não-codificado de mesma eficiência espectral do sistema com codificação wavelet $(1 \mathrm{bit} / \mathrm{s} / \mathrm{Hz})$, utilizado apenas como referência de desempenho para o canal com desvanecimento Rayleigh plano.

Dentre os sistemas simulados, aquele que apresentou o melhor desempenho possui perfil de intensidade de múltiplos percursos dado pelo canal 1 (ver Tabela II). Essa configuração do canal representa um canal com desvanecimento plano, logo não há interferência intersimbólica, uma vez que a atenuação do canal afeta o símbolo transmitido somente durante um período de amostragem. 


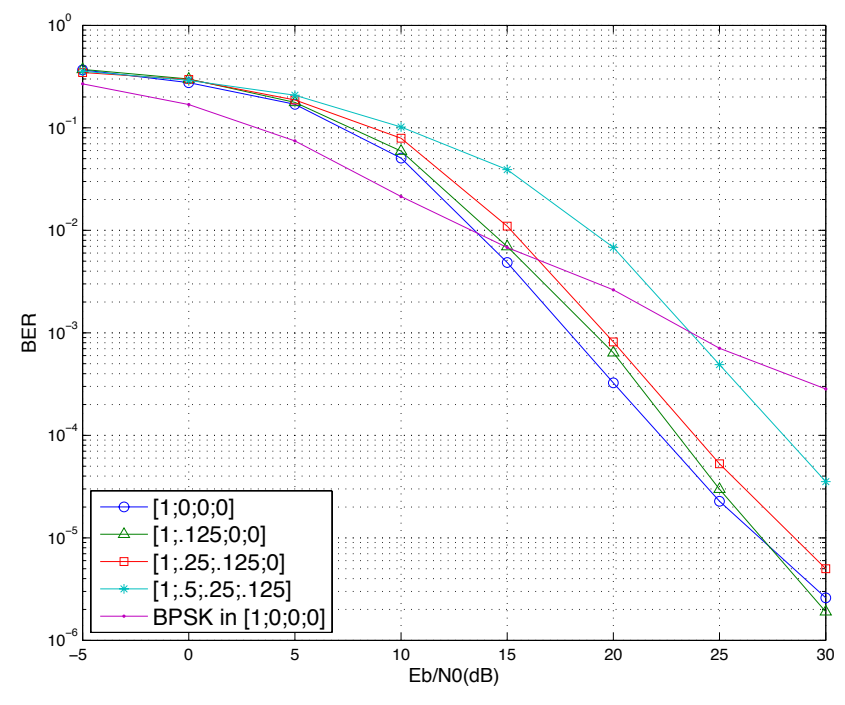

Fig. 4. Probabilidade de erro de bit para os sistemas analisados.

Existe um aumento natural na taxa de erro do sistema codificado com perfis de intensidade dados pelos canais 2 , 3 e 4 (ver Tabela II). Este último perfil modela um canal fortemente seletivo em frequência. Isto se deve graças à pequena diferença entre os valores de potência média dos primeiro e segundo perfis, principalmente, mas também pelo maior alastramento de símbolos transmitidos em determinados instantes ao longo de intervalos de sinalização consecutivos. Nessa configuração, os quatro perfis de potência média por atraso se encontram ativos, o que implica na influência de um sinal durante três intervalos de sinalização, influenciando a energia de três sinais adjacentes.

De acordo com o gráfico da Figura 4, para a faixa de $E_{b} / N_{0}$ entre $-5 \mathrm{~dB}$ e $13 \mathrm{~dB}$, os sistemas codificados apresentam desempenho inferior ao do sistema de referência. Isto se deve ao número de símbolos da constelação 11-PSK adotada pelo sistema codificado. Os baixos valores de SNR intensificam os erros de demodulação entre os símbolos da constelação 11-PSK, enquanto que na constelação BPSK, os sinais antipodais se mostram mais robustos aos efeitos do canal sem fio. Nesse caso, o ganho de diversidade conseguido com a codificação wavelet não reverte a perda de desempenho motivada pelos erros de demodulação.

A partir de $15 \mathrm{~dB}$ o sistema codificado já apresenta ganhos de desempenho, inclusive em um ambiente de comunicação sujeito a seletividade em frequência. Em valores de BER próximos a $10^{-4}$, o sistema codificado, sujeito a seletividade em frequência, apresenta ganho de desempenho superior a 10 $\mathrm{dB}$ em relação ao sistema BPSK sujeito ao desvanecimento plano.

Por fim, é importante ressaltar que a codificação wavelet se mostrou robusta aos efeitos provocados pela interferência intersimbólica de canais seletivos. Em níveis moderados de seletividade, o desempenho do sistema codificado é muito próximo daquele obtido em canais planos. Essa robustez da codificação wavelet, frente aos efeitos seletivos de alguns canais sem fio, habilita o uso da técnica em diferentes cenários de comunicações.

\section{CONCLUSÕES}

Neste artigo, o desempenho da codificação wavelet, em termos da probabilidade de erro de bit, foi avaliado em um sistema de comunicação sujeito ao desvanecimento Raleigh variante no tempo e seletivo em frequência. Resultados numéricos, obtidos por simulação computacional para diferentes perfis de intensidade de potência, demonstram a eficiência desta técnica de codificação na minimização dos efeitos destrutivos desses canais sobre a informação transmitida.

\section{AgRADECIMENTOS}

Os autores Lucas Cavalcante e Aluísio Rêgo contam com o suporte financeiro do Programa de Apoio de Reestruturação e Expansão das Universidades Federais (REUNI) e da Coordenação de Aperfeiçoamento de Pessoal de Nível Superior (CAPES), respectivamente.

\section{REFERÊNCIAS}

[1] T. S. Rappaport, Wireless Communication: Principles and Practice, 2nd ed. New Jersey: Prentice Hall, Dezembro, 2001, no. ISBN 0130422320 .

[2] W. H. Tranter, K. S. Shanmugan, T. S. Rappaport, and K. L. Kosbar, Principles of Communication Systems Simulation with Wireless Applications. USA: Prentice Hall, 1st ed., 2003.

[3] M. C. Jeruchim, P. Balaban, and S. K. Shanmugan, Simulation of Communication Systems. New York: Kluwer Academic / Plenum Publishers, 2nd ed., 2000.

[4] M. Pätzold, Mobilfunkkanäle (Mobile Fading Channels). Wiesbaden: Braunschweig, 1st ed., 1999.

[5] B. Sklar, "Communication engineering services; rayleigh fading channels in mobile digital communication systems part i: Characterization," IEEE Communications Magazine, Julho, 1997.

[6] B. Sklar, "Communication engineering services; rayleigh fading channels in mobile digital communication systems part ii: Mitigation," IEEE Communications Magazine, Julho, 1997.

[7] H. Resnikoff and R. Wells, Wavelet analysis: the scalable structure of information, 1st ed., ser. ISBN 0-387-98383-X, Verlag, Ed. Springer, 1998.

[8] M. A. Tzannes and M. C. Tzannes, "Bit-by-bit channel coding using wavelets," Proceedings of IEEE GLOBECOM'92, pp. 684-688, Abril, 1992.

[9] L. F. Q. Silveira, L. G. Silveira, F. Assis, and E. L. Pinto, "Analysis and optimization of wavelet-coded communication systems," IEEE Transactions on Wireless Communication, vol. 8, no. 2, pp. 263-267, Fevereiro, 2009.

[10] L. G. Silveira, L. F. Q. Silveira, and F. Assis, "Performance analysis of space diversity scheme in wavelet-coded systems over fading channels," in International Microwave \& Optoelectronics Conference, no. 978-1-4244-5357-3. SMBO/IEEE MTT-S, 2009, pp. 277-281.

[11] L. F. Silveira, F. Assis, and E. L. Pinto, "Performance of a psk system with wavelet time diversity over flat rayleigh fading channels," Proceedings SBMOIIEEE MTT-S IMOC, no. 0-7803-7824-5, pp. 187-191, 2003.

[12] L. G. Silveira, L. F. Q. Silveira, F. Assis, and E. L. Pinto, "Soft-Decision Demodulation of Wavelet-Coded PSK Signals over Flat Rayleigh Fading Channels," in ITS'2006 - VI International Telecommunications Symposium, Setembro, 2006, Fortaleza-CE.

[13] L. F. Silveira, "Análise da Codificação Wavelet em Sistemas Sujeitos ao Desvanecimento Rayleigh Plano," in Tese de Doutorado/ DEE - UFCG, Junho, 2006.

[14] J. G. Proakis, Digital Communications, 2nd ed. New York: McGraw-Hill, 1989, no. ISBN 0070509379.

[15] B. Sklar, Digital Communications: Fundamentals and Applications, 2nd ed. New York: Prentice Hall, Janeiro, 2001, no. ISBN-10 0130847887. 